\title{
Uso clínico de corticosteróides em endodontia
}

\section{Clinical use of corticoesteroids in endodontics}

Elaine Vianna Freitas Fachin* Andréa Soares Lubaszewski** Maria Elena Gageiro Soares** Marco Antônio Acosta Bisognin** Nicolau Fonseca Milano*

\section{UNIVERSIDADE FEDERAL DO RIO GRANDE DO SUL FACULDADE DE ODONTOLOGIA BIBLIOTECA}

\section{Resumo:}

\begin{abstract}
Estudo "in vivo" $\mathrm{e}$ "in vitro" utilizando beta metasona (Celestone) como medicação intra canal. Clinicamente observou-se ausência de sintomas após a utilização do medicamento em casos de biopulpectomia. No estudo "in vitro" foi verificada a penetraçå a nível apical do corticoesteroide nos dentes extraídos das duas arcadas. sendo que mais notadamente nos dentes inferiores.
\end{abstract}

\section{Summary:}

"In vivo" and "in vitro" study in which beta methasone (Celestone) was used as intracanal medicament. Clinically, there were no symptoms after its use in cases of pulpectomy. Corticoesteroid apical penetration was observed in extracted teeth, most markedly in the lower dental arch.

\section{Unitermos:}

Endodontia, Curativo de Demora, Corticoides, Canal radicular-terapia.

\section{Introdução:}

C orticoesteroides tem sido largamente utilizados em Endodontia somo medicação intra canal $2,7,5,6,8,9,10,11,12,13,15$. Atuam como potentes antiinflamatórios controlando a reação tecidual pós-operatória em dentes com polpa viva submetidos a procedimentos conservadores do tipo pulpotomia ou a procedimentos radicais como biopulpectomias $3,5,6,11$.

As respostas teciduais decorrentes de intervenções pulpares conservadoras ou radicais incluem: vasodilatação, aumeno da permeabilidade capilar e edema. Os fenômenos vasculares e celulares, dentro dos diminutos espaços do canal radicular podem ocasionar dor.

Após biopulpectomias, a dor pode surgir como sequela indesejável da inflamação decorrente da extirpação da polpa ou, no caso de tratamentos conservadores pulpares, da remoção parcial da polpa. No entanto, a sintomatologia dolorosa é provocada, mais freqüentemente, por manobras técnicas agressivas como sobreinstrumentaçăo e uso de solu. ções irrigadoras irritantes que agravam a reaçăo tecidual apical.

Alguns trabalhos $6,8,9,15$ descrevem sucesso clínico e histológico após uso tópico de pomadas e cremes com corticóides em curativos de demora nos casos de biopulpectomias. Outros estudos ${ }^{10,12}$ utilizam associações de corticoides e antibióticos sob forma de cremes dermatológicos com a finalidade de controlar a inflamação pós pulpectomia e combater eventuais microorganismos do canal radicular. Sabe-se, no entanto, que em casos de polpa viva a presença de flora microbiana é mínima ou inexistente, fato esse que descarta, em tese, a necessidade de antibióticos. Dessa maneira, parece lícito investigar-se outra forma de apresentação de corticoides para uso endodôntico.

$O$ presente estudo tem as seguintes proposições: $1{ }^{\text {a }}$ ) verificar a sintomatologia clínica de dentes com polpa viva submetidos a tratamento endodôntico medicados com solução aquosa de betametasona (colírio). 2.) avaliar "in vitro" a penetraçăo desse medicamento dentro do canal radicular.

\section{Materiais e metodos:}

estudo "in vivo" foi desenvolvi do no Curso de Especializaçăo

\footnotetext{
* Professores de Endodontia FO/UFRGS

** Alunos Curso de Especialização em Endodon tia FO/UFRGS
}

\begin{tabular}{|l|l|l|l|l|l|l|}
\hline R. Fac. Odontol. & Porto Alegre & V.34 & N.1 & p.19-22 & SETEMBRO & 1993 \\
\hline
\end{tabular}


em Endodontia da FO-UFRGS. Casos clínicos de polpa viva receberam, após preparo químico mecânico e secagem, a solução aquosa betametasona (Celestone - Schering) como medicaçăo intracanal.

Nos casos de biopulpectomia introduziu-se de 1 a 3 gotas da solução de betametasona na entrada do canal radicular, levadas com o auxílio dos mordentes da pinça odontológica. Após, o medicamento foi levado ao interior do canal radicular com o instrumento memória com movimentos de introdução e traçăo. Ao final do tratamento, as respostas clínicas foram anotadas em ficha individual com os seguintes itens:

a) Motivo da consulta - queixa atual, diagnóstico, retratamento endodôntico, biopulpectomia ou necrose pulpar.

b) Dor pré-operatória (sintomático ou assintomático). Se sintomático, descreva a dor quanto ao tipo, intensidade, localização e duração.

c) Dor pós-operatória significativa?

Para o estudo "in vitro" foram utilizados 30 dentes monorradiculares extraídos, divididos em 2 grupos:

Grupo 1: 15 dentes anteriores superiores.

Grupo 2: 15 dentes monorradiculares inferiores.

Todos os dentes extraídos receberam preparo químico mecânico com a técnica escalonada ${ }^{14}$. Padronizouse como instrumento memória a lima tipo Kerr $40 \mathrm{com}$ alargamento cervical progressivo até a lima tipo Kerr 60 e irrigaçăo simultânea com hipoclorito de sódio a $1 \%$.

Após o preparo, os canais foram secados com papel absorvente e pingou-se de 1 a 3 gotas da solução de betametasona na entrada do canal radicular com o auxílio dos mordentes da pinça odontológica. Assim como no estudo "in vivo", o medicamento foi levado ao interior do canal radicular com o instrumento memória com movimentos de introdução e tração.

Para visualização da penetração do medicamento na região apical, foi feito preparo apical nos dentes extraídos com broca de aço esférica $1 / 2$ seguindo orientação paralela ao longo eixo do dente. Foi confeccionado um tampão apical com cone de papel absorvente penetrando $3 \mathrm{~mm}$ dentro do canal radicular (Figura 1). Para fins de contraste, pingou-se 1 gota do corante azul de metileno a $2 \%$ em $5 \mathrm{ml}$ do medicamento. Após o preparo, os dentes foram fixados através da coroa em tiras de cera utilidade de acordo com a Figura 2.

\section{Resultados:}

A análise da sintomatologia foi feita a partir do somatório das respostas individuais dos pacientes testados. A análise visual da penetraçăo "in vitro" foi feita a partir da marcação do corante no tampão apical de papel absorvente.

O resultado clínico mostrou que de 13 casos com medicação, apenas um apresentou sintomatologia pós-operatória. Esse resultado indica que $92,3 \%$ dos pacientes testados apresentaram ausência de sintomas após utilização da Solução de Betametasona (Celestone) como curativo de demora em biopulpectomias.

A observação visual, realizada por 3 observadores, apresentou os resul- tados descritos na Tabela 1 (dentes inferiores) e na Tabela 2 (dentes superiores). A observação visual baseou-se nos seguintes critérios: (0) tampão apical seco, $(+)$ tampão apical molhado $1 \mathrm{~mm},(++)$ tampão apical molhado mais de $1 \mathrm{~mm}$.

\section{FIGURA 1}

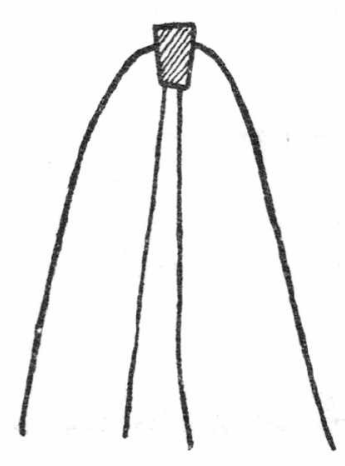

Esquema de corte longitudinal da raiz. Detalhe tampão apical de papel absorvente

\section{Figuea 2}

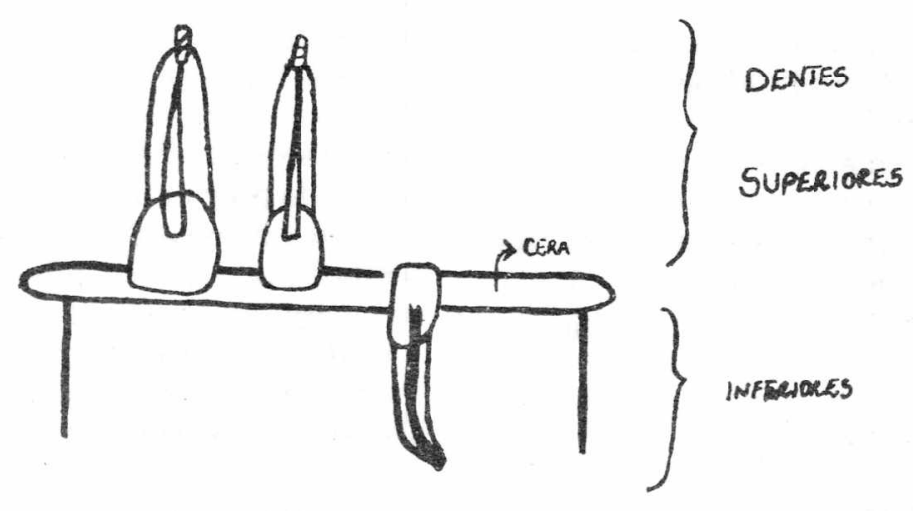

Esquema da posiça dos dentes sobre cera utifidade apoiados em cubeta de vidro

R. Fac. Odontol.

Porto Alegre

V. 34

N. 1

p.19-22

SETEMBRO

199ః 


\section{Tabela 1}

Penetraçăo "in vitro" da solução aquosa de betametasona nos dentres inferiores

\begin{tabular}{ccc} 
Dente & $\begin{array}{c}\text { Compr. } \\
\text { Trabalho }\end{array}$ & $\begin{array}{c}\text { Penetra- } \\
\text { ção }\end{array}$ \\
\hline
\end{tabular}

1 Prémol. $16 \mathrm{~mm} \quad++$

2 Prémol. $16 \mathrm{~mm}+$

1 Prémol. $19 \mathrm{~mm}++$

1 Prémol. $18 \mathrm{~mm} \quad++$

2 Caninos $21 \mathrm{~mm} \quad++$

1 Canino $19 \mathrm{~mm} \quad++$

1 Canino $22 \mathrm{~mm} \quad++$

1 Canino $17 \mathrm{~mm}+t$

2 Caninos $20 \mathrm{~mm} \quad++$

1 Canino $21 \mathrm{~mm}+t$

1 Canino $22 \mathrm{~mm} \quad++$

1 Canino $23 \mathrm{~mm} \quad++$

\section{Tabela 2}

Penetração "in vitro" da solução aquosa de betametasona nos dentes superiores

\begin{tabular}{llc}
\hline Dente & $\begin{array}{c}\text { Compr. } \\
\text { Trabalho }\end{array}$ & $\begin{array}{c}\text { Penetra- } \\
\text { ção }\end{array}$ \\
\hline
\end{tabular}

\begin{tabular}{lll}
1 Inc. Cen. & $17 \mathrm{~mm}$ & ++ \\
1 Inc. Cen. & $15 \mathrm{~mm}$ & ++ \\
1 Inc. Cen. & $19 \mathrm{~mm}$ & + \\
1 Inc. Cen. & $17 \mathrm{~mm}$ & + \\
1 Inc. Cen. & $20 \mathrm{~mm}$ & + \\
2 Inc. Cen. & $19 \mathrm{~mm}$ & 0 \\
1 Inc. Cen. & $17 \mathrm{~mm}$ & 0 \\
1 Inc. Cen. & $17 \mathrm{~mm}$ & + \\
1 Inc. Cen. & $15 \mathrm{~mm}$ & + \\
2 Inc. Cen. & $19 \mathrm{~mm}$ & ++ \\
1 Inc. Cen. & $18 \mathrm{~mm}$ & ++ \\
1 Inc. Cen. & $18 \mathrm{~mm}$ & + \\
1 Inc. Cen. & $20 \mathrm{~mm}$ & + \\
\hline
\end{tabular}

\section{Discussão:}

A utilização da Solução de Betametasona na forma de colírio (Celestone) como medicação de demora, em casos de biopulpectomia, a exemplo de outros corticoesteroides, apresentou bons resultados clínicos já que, de todos os casos testados, em apenas um ocorreu sensibilidade dolorosa depois da utilização do medicamento. Esses achados estão de acordo com resutlados de outras investigações $2,6,8,9,13,15$ que relatam diminuição de sintomatologia pós-operatória em Endodontia. De acordo com FACHIN, E.F. ${ }^{4}$ a betametasona apresentou efeito antiinflamatório mais potente que a triancinolona e hidrocortisona, limitando a chegada de neutrófilos e estabilizando as membranas das lisosomas do tecido pulpar após pulpotomia. Os efeitos observados são explicados por LUYK et al. ${ }^{7}$ ao listar o mecanismo de ação dos corticoesteoirdes: a) redução do infiltrado inflamatório e produtos celulares; b) efeitos sobre a produção de anticorpos, quininas e prostaglandinas; c) vasoconstrição na área inflamatória que diminui a saída de líquido aos tecidos, prevenindo edema e desconforto, d) estabilização das membranas das lisosomas que inibe a saída de enzimas hidroliticas responsáveis pelo processo inflamatório.

A metodologia utilizada no presente estudo foi adequada para a verificação dos objetivos propostos. No experimento "in vitro", a despeito de os dentes estarem mantidos em temperatura ambiente, onde a dentina desidratada apresenta absorção do medicamento diferente da situação "in vivo", a penetração da solução até o foramem aplical, foi medida em $90 \%$ dos dentes. Esse resultado, evidencia "in vitro" o contato do medicamento com as estruturas apicais e mostra que a técnica da introdução da solução com os mordentes da pinça, associada ao uso do instrumento com movimen- tos de introdução e tração foi efetiva.

O tampão apical de cone de papel pretendeu simular a presença do coto pulpar. Dessa forma, o medicamento junto ao corante, tingindo o tampão de papel, representa a chegada do medicamento ao coto pulpar. Esse fato é de particular importância já que o medicamento para atuar deve ter contato direto com o tecido conjuntivo pulpar.

Mantendo os dentes superiores com o foramem para cima e os inferiores com o foramem para baixo, observou-se significativa diferença no grau de penetração do medicamento. A ação da força da gravidade facilita a chegada da solução na região apical dos dentes inferiores. Em alguns casos, o simples pingar do medicamento com a pinça, sem a ação do instrumento, já foi suficiente para a umectação apical. Nos dentes superiores, a penetração foi nitidamente menor, e em alguns casos inexistente.

Esses achados podem ser transpostos em maior ou menor grau para a situação clínica, levando-se em conta, no entanto, que "in vivo" a pressão negativa dos tecidos periapicais possa ser maior e dificultar a chegada do medicamento ao ápice do que a pressão do tampão de papel utilizada no experimento "in vitro".

Sugere-se que "in vivo", nos dentes superiores, se utilize mais gotas do medicamento e que se permaneça mais tempo na ação de introdução e tração da lima para que o medicamento chegue ao ápice.

A apresentação do medicamento na forma de colírio, contendo apenas a solução de corticoesteroide, apresenta duplo destaque: ser utilizado em tecidos de origem semelhante ( tecido conjuntivo dos olhos e tecido conjuntivo pulpar) e deixar livre e mais concentrada a ação do corticoesteroide sem combinação com antibiótico.

Dessa forma, o curativo de demora com antibiótico descrito por PAlVA, J.G. ${ }^{10}$, nos casos de biopulpectomias, pode ser substituído com 
vantagem pelo uso de corticoesteroides 4,5 , uma vez que 0 componente microbiano nesses casos não é significativo. Além disso, as associaçð̄es antibiotico/corticoesteroide (Rifocort), apesar de apresentarem bons resultados clínicos, são medicamentos indicados para uso dermatológico e não para uso sobre a polpa dentária. Sobre o tecido conjuntivo pulpar, estariam melhor indicados colírios por atuarem sobre tecidos de origem semeIhante. $O$ teste de medicamentos sobre o olho do coelho, segundo BOMBANA, et alii ${ }^{1}$, constitui-se num dos mais usados na comprova- ção do potencial inflamatório de fármacos de uso endodôntico.

Acresce-se que a betametasona, presente no colírio (Celestone), é farmacologicamente mais potente ${ }^{7}$ que a prednisolona presente nos cremes dermatológicos (Rifocort).

Finalmente, quanto ao uso clínico da solução, por não conter gorduras, apresenta facilidade de remoção das paredes do canal quando comparada aos cremes dermatológicos.

\section{Conclusões:}

1. A solução aquosa de betametasona (Celestone) apresenta-se como alternativa de medicação intracanal após biopulpectomias.

2. A betametasona sem combinação com antibiótico, utilizada como curativo de demora, apresentou ausência de sensibilidade dolorosa em $92,3 \%$ dos casos.

3. Foi evidenciada "in vitro" a penetração apical do medicamento em $90 \%$ dos dentes testados.

4. A penetração do medicamento foi maior nos dentes inferiores do que nos superiores.

\section{Referências Bibliográficas}

1. BOMBANA, A.C.; PAIVA, J.G.; ALVARES, S.; ANTONIAZZI, J.H. Reação Inflamatória do olho de coe tho que se segue à instilação de alguns fármacos de uso endodôntico. Rev. Ass. Paul. C. Dent., Săo Paulo, v. 28, n? 4, p. 216-223, jul/ ago. 1974.

2. CHANCE, K.; LIN, L.; SKRIBNER, J. Corticosteroid use in acute apical periodontitis: a review with clinical implications. Clin Prev Dent., Waco Tx, v. 10, p. 7-10, 1988.

3. CLAMAN, H.N. How corticoesteroids work. J. Allergy Clin Immunol., St. Louis, v. 55, p. 145-151, 1975.

4. FACHIN, E.F. Effects of Steroids on postsurgically inflamed dental pulp. A Histological and Cytochemical Study. Chicago, 1989. Tese para obtenção do trtulo de Master of Science pela Universidade de Illinois em Chicago.

5. FACHIN, E.F.; ZAKI, A.E. Histology and lysosomal cytochemistry of the post surgically inflamed dental pulp after topical application of steroids. I. Histological sudv. J. of Endo.,
Baltimore, v. 17, n. 9, p. 457-460, 1991.

6. LANGELAND, K.; LANGELAND, L.K. and ANDERSON, D.M. Corticoesteroids in dentistry. 'Biological effects of corticoesteroids in pulp and periapical tissues. Int. Dent. J., Guildford, v. 27, p. 217-251, 1977.

7. LUYK, N.H.; ANDERSON, J.; WARDBOOTH, R.P. Corticosteroid The rapy and the Dental Patient. Brit Dent J., London, v. 159 , n: 1, p. 12 17, 1985

8. MOSTELLER, J.H. The ability of a prednisolone solution to eliminate pulpa inflamation. J. Prosth. Dent., St. Louis, v. 13 , n. 4 , p. $654-660$, jul/ ago 1963

9. KRASNER, P.; JACKSON, E.:Manage ment of posttreatment endodontic pain with oral dexamethasone.: a double blind study. Oral Surgery. St. Luis, v. 62, p. 187-190, 1986.

10. PAIVA, J.G. Contribuiçăo ao Estudo experimental da medicaçăo pbspulpectomia. 0 uso de uma associaçåo corticoesteroide/anbibibtico como curativo de "demora". Såo paulo, 1969. Tese para a obtenção do título de doutor pela Faculdade de Odontologia - USP.

11. STANLEY, H.R. Questions, answers and lingering controversies. In: Co hen, S.; Burns, R.C. Pathways of the pulp, St. Louis, Mosby, p., p. 709-710, 1980

12. VAN CURA, J. and REMEIKIS, N. Corticoesteroid-Antibiotic combination in the treatment of acute secondary periodontites. Int Dent J., Guildford, v. 39, p. 307-312, 1970.

13. VARELA, J.A.F. et alli. Prevençå das sequelas pós pulpectomia. Rev. Fac. Odont., S. Paulo, v. 3, no 1, p. 201-210, jan/jun 1962.

14. WEINE, F.S., HEALEY, H.J., GERSTEIN, H., EVANSON, L. Precurved files and incremental instrumentation for root canal enlargement. J. Can. Dent. Ass., Ottawa, v. 36, n: 4, p. 155-7, 1970.

15. WOLFSON, B. The role of hydrocortisone in the control of apical periodontites. Oral Surg Oral Med Oral Pathol., St. Louis, v. 7, n. 314-321 1954. 